\title{
Notions such as "truth" or "correspondence to the objective world" play no role in explanatory accounts of perception
}

\author{
Rainer Mausfeld
}

Published online: 18 September 2015

(C) Psychonomic Society, Inc. 2014

\begin{abstract}
Hoffman, Singh, and Prakash (Psychonomic Review and Bulletin, 2015, in press) intend to show that perceptions are evolutionarily tuned to fitness rather than to truth. I argue, partly in accordance with their objective, that issues of 'truth' or 'veridicality' have no place in explanatory accounts of perception theory, and rather belong to either ordinary discourse or to philosophy. I regard, however, their general presumption that the evolutionary development of core achievements of the human perceptual system would be primarily determined by aspects of fitness and adaption as unwarranted in light of the evidence available.
\end{abstract}

Keywords Visual perception · Concepts · Mathematical models

In our ordinary intuitions about perception, we are convinced that our perceptual apparatus by and large conveys to us a basically 'truthful' account of the external world. We are apparently biologically endowed with a predisposition to accept certain expressions generated by our perceptual capacities for 'things in the objective world.' This propensity constitutes a kind of naïve realism that is deeply built into our mental make-up (cf. Mausfeld, 2010a). Although naïve realism already founders in the face of most elementary scientific facts, for instance about the properties of our sense organs, it intellectually expresses some of our deepest convictions about the mental activity of perceiving, namely being in direct touch with a mind-independent world. These convictions are so deeply entrenched in our conception of the world and our interactions with it that it is hardly surprising that they exercise a continuous impact on perception research. Attempts to cope with them pervade the entire history of rational enquiry into

R. Mausfeld $(\bowtie)$

Institute for Psychology, University of Kiel, D-24098 Kiel, Germany

e-mail: mausfeld@psychologie.uni-kiel.de perception since the time of the Pre-Socratics. Variants of more refined forms of naïve realism are still prevailing as a tacit background assumption in current perceptual psychology, albeit often concealed by technically sophisticated formulations.

Hoffman, Singh and Prakash (HSP) set out to mount a new attack on widely held convictions in perceptual psychology according to which perception somehow mirrored structural aspects of the 'objective world.' HSP approach this issue from a new direction, namely through a kind of conceptual analysis based on mathematical techniques from evolutionary game theory and genetic algorithms. They formulate two questions that they want to answer in the paper. "First, is the vocabulary of our perceptions isomorphic to aspects of objective reality so that our language of perceptions could, in principle, describe the objective truth? Second, if so, do our perceptual systems in fact succeed in describing the true state of affairs in the world?" The main thrust of their paper is to demonstrate by mathematical inferences from allegedly rather simple premises that natural selection "shapes perceptions to guide adaptive behavior." According to HSP, the "key insight from these evolutionary games" is that "natural selection tunes perception to payoffs, not to truth."

In this comment, I will focus on two issues: (1) I will try to locate HSP's endeavor within the intellectual landscape of the history of perception theory. (2) I will deal with the specific premises, in particular regarding 'truth' and 'objective world', which they employ as starting points for their mathematical analyses, and bring into question, for the explanatory purposes of perception theory, both these premises and the types of mathematical idealizations associated with them.

Perception theory, with its history of about 2,500 years, belongs to the oldest fields of psychology and in fact science. Although our theoretical understanding of the principles on which the achievements of the perceptual system are based still barely scratches the surface, important theoretical insights 
have been achieved in the course of these enquiries that no contemporary explanatory account should ignore. All the same, current orthodoxy in perceptual psychology has profoundly fallen back behind theoretical insights previously achieved. These regressions in theoretical understanding seem to be predominantly due to the detrimental influences of tacit commonsense conceptions with respect to perception (cf. Mausfeld, 2011) as well as with respect to core methodological principles of the natural sciences (cf. Mausfeld, 2012). HSP provide ample evidence from the perception literature for such theoretical distortions due to commonsense intuitions, at the core of which is the idea that the 'objective world' is somehow mirrored in perception.

Already at the very beginning of the scientific enterprise, important strands in ancient Greek philosophy were guided by the conviction that the senses could not discover truths about the physical world. Early enquiries into the nature of perception, albeit mostly in theological and metaphysical contexts, reached their first culmination point in the 13th century (prominently Aquinas, Scotus, Ockham), where the possibility that "perceptions of extramental reality were not only sometimes, as in the case of sensory illusions, but inevitably inaccurate" (Tachau, 1988, p. 69), received intense theoretical treatment. In the wake of the 'Galilean revolution', these enquiries into the nature of perception came to be progressively transformed into what, in modern terms, is regarded as a natural science framework, and reached their second culmination point during the 17th century in what is sometimes referred to as the 'first cognitive revolution.' For instance Cudworth, closely following Descartes, expressed in his Treatise of 1731 his conviction that "sense, which either lies in the same level with that particular material object which is perceived, or rather under it and beneath it, cannot emerge to any knowledge or truth concerning it." Yolton (1984, p. 222) noted in his seminal treatment that "the majority of seventeenth- and eighteenthcentury writers did not consider the mind to be a mirror of nature". In the 19th century, Müller's famous Law of Specific Nerve Energy "contains an explicit denial of any necessary qualitative connection or resemblance between the physical processes of stimulation and the psycho-physiologic changes associated therewith in the sense-organ and "consciousness" (Brett, 1921, p. 109). These are but a few examples from the intellectual tradition of perception theory, whose core insights were, mostly implicitly, echoed in Gestalt psychology and ethology. These insights had to be achieved in long and arduous attempts to break away from Aristotelian conceptions of perception (e.g. King, 1994), which were guided and moulded by commonsense intuitions about perception.

Aristotelian conceptions of perception were premised on the idea that external physical objects and perceptual objects are commensurable through a similarity relation, and that perception basically amounts to a process of increasing abstraction which transforms sensory information into perceptual categories. In contrast, the reconceptualizations of theoretical enquiries into perception that were achieved during the 17 th century are tantamount to a radical break with pictorial or similarity-based conceptions of perception. It became increasingly clear that the explanatory gap between what is provided by the senses on the one hand, and what is achieved by the perceptual system on the other, cannot be bridged within our ordinary conceptualizations of perception, which regard the core perceptual relation as going inward from the senses. Descartes, within a genuine natural science approach, understood that the core explanatory relation between external physical events and conceptual forms (or 'perceptual ideas' in the terminology of the time) has to be reversed. Rather than construing it as going inward from the senses, this relation has to be understood as going outward, as it were, from a predetermined system of conceptual forms. The sensory signs receive the triggering capacities that they have through the specific nature of the given conceptual forms rather than because of their respective physical causes in the external world. Or, as Cudworth put it: "Sense is but the offering and presenting of some object to the mind, to give it an occasion to exercise its own inward activity upon." Descartes accordingly emphasized, e.g. in his Principles of Philosophy of 1644, that our perceptions do not teach us "what is really in things, but merely that whereby they are for us useful or hurtful" ("quid ad illam externa corpora prodesse possint, aut nocere") - in HSP's formulations, perceptions are tuned to fitness rather than truth. These enquiries brought forth a sophisticated form of 'sign conception' of the basic mode of operation of the perceptual system (see Yolton, 1996, p. 183ff., and MeierOeser, 1997, p. 350ff., for illuminating treatments). Unfortunately, however, these theoretical insights into what might be regarded as the Fundamental Problem of perception theory namely the identification of the abstract principles by which the perceptual system, regarded as a specific biological subsystem of the mind/brain, can generate, from specific physicogeometrical energy pattern, its output - sank entirely into oblivion.

As a result, the previously achieved insights into the logical gap, and hence the incommensurability, between categories of the 'objective world' and perceptual categories, and into the nature of a mediating operation, had not been given an opportunity to find an appropriate formulation within the conceptual framework that became available due to developments in mathematical logic and the theory of computational systems. In modern terms, there is a 'type mismatch' between structural properties and types of individuation that objects of the 'objective world' have and those that 'perceptual objects' have. Perceptual predicates can neither be defined by sensory predicates nor by predicates in terms of which we describe the mind-independent 'physical world.' Hence, the type of primitives underlying 'perceptions' - including core ones such as 'Gestalt' or 'perceptual object' - cannot be derived, 
by whatever kind of inductive mathematical machinery, from the sensory input, or, more generally, from experience (cf. Mausfeld, 2010a, 2013).

A second important theoretical insight was achieved during the 'first cognitive revolution.' If we investigate, within a natural science perspective, modes of operations of our mind, we have to take into account that we are dealing with a compound system that is organized in the form of an entire orchestra of subsystems, each of which has its proprietary primitives and modes of operation. Hence, when we are theoretically dealing with perception, we have to overcome our ordinary conviction that the appropriate unit of analysis is that of a 'person.' Rather, we have to think in terms of a mental architecture (for medieval insights into this problem, see, e.g., King, 2008), and distinguish, for instance, systems that deal with perception from, say, interpretive, linguistic, or motorial systems that take advantage of the output of the perceptual system. By taking into account the functional architecture into which the perceptual system is embedded, it came to be understood that notions such as 'error', 'veridicality', or 'illusion' have no place in explanatory accounts of the perceptual system because they cannot be ascribed to the perceptual system proper, but rather pertain to a different unit of analysis, namely that of a person. For instance, Arnauld and Nicole stated in their Port-Royal Logic of 1642 "that there can be no illusion or error" in perception, and that "the whole error solely results from our false judgments." In the same vein, Kant in his Anthropology remarked: "The senses do not deceive us...because they do not make judgements at all, and that is the reason why the error always is due to the intellect." And, similarly, Helmholtz: "The senses cannot deceive us, they work according to their established immutable laws and cannot do otherwise. It is us who are mistaken in our apprehension of the sensory perception." Similar insights have been expressed by, for example, Hering, Koffka, or Köhler (see Mausfeld, 2002, 2010a, b, 2011, for references to the works cited). The pre-occupation with 'perceptual illusions,' which is still characteristic for large fields of perceptual psychology and philosophy of perception, indicates the extent to which perceptual research is still driven by commonsense conceptions of perception.

In the natural sciences, we would succumb to an unjustifiable "methodological dualism" (Chomsky, 2000) if we allow our enquiries of human biological systems 'above the neck' to be guided by different methodological principles than those that we employ for those 'below the neck.' Such methodological considerations already make it highly doubtful that notions such as 'veridicality' or 'correspondence to the objective world' would play any role in explanatory accounts of the structural and computational properties on which the achievements of our perceptual system are based. To be sure, our built-in conviction that perception represents the way the external world is, constitutes itself a functionally important achievement of our mind/brain, and thus ultimately has itself to find a place in an explanatory account of our mental capacities. It becomes detrimental, however, when it is, with respect to perception theory, illegitimately transferred to scientific enquiry.

So, even a marginal consideration of what has been achieved during the long history of naturalistic enquiry into the nature of perception should make clear that issues of 'veridicality' or of a 'correspondence to the objective world' have, in the course of corresponding theoretical developments, been recognized as irrelevant for the explanatory purposes of perception theory. Hence, there is (or at least should be) no target left for the main thrust of HSP's arguments. But sadly enough, the actual state of affairs in perceptual psychology testifies to the contrary. This is due to the disregard or even disdain that current orthodoxy in perceptual psychology seems to show for previously achieved theoretical insights. In the wake of empiricist and behavioristic conceptions of mind, perceptual psychology is still predominantly under the influence of basically Aristotelian conceptions of perception (even if this fact is often veiled by highly sophisticated technical vocabulary).

Given the present state of the field, one might consider it reasonable to develop new kinds of arguments by which perceptual psychologists could be convinced that, for the explanatory goals of perception theory, their intuitions about 'correspondences to the objective world' or about 'veridicality' are profoundly inappropriate. This is what HSP intend to show by approaching the issue from a new direction. Whether their approach is a suitable one for achieving their objective depends above all on whether the specific premises on which their mathematical analyses rest are sufficiently clear and coherent. I will very briefly critically discuss these premises, first from within their own framework, then from a more general perspective.

HSP base their analysis on a notion of 'objective world,' whose meaning they apparently presume to be self-evident and a matter of course. They also take this meaning to be precise enough to be mathematically idealizable in terms of a set $W$ and a $\sigma$-algebra $\mathrm{W}$ over $W$, yielding measurable events (which they need in order to get the probability spaces underlying their dynamic models). Accordingly, they formalize the 'objective world' by a measurable space $(W, \mathrm{~W})$. Analogously, they formalize "possible perceptual experiences of an organism by a measurable space $(X, \mathrm{X})$, where $X$ is a set of possible experiences and $\mathrm{X}$ is a collection of subsets of $X$ called events." Sometimes and apparently synonymously, HSP also refer to $X$ as "perceptual representations," and tentatively interpret $X$ as "the possible phenomenal states of the observer."

The force of their arguments and the validity of their conclusions naturally rest on the presupposition that we have a clear understanding of what precisely $W$ and $X$ are. I must 
admit, however, that the conceptualization of both $W$ and $X$, and of their relation, remains opaque to me. These basic notions are, even if they might receive a deceptive gloss of obviousness, highly technical notions in the present context. Their precise meaning thus cannot be tacitly presupposed. Here, I can only hint at some of the issues that seem to me to require clarification.

HSP seem to take the "objective world $W$ " as consisting of "states of the world." As these states are regarded as 'objective,' one might, in one interpretation of the term, take this to mean that they do not change with 'perspective,' sufficiently abstractly conceived (as, for instance, in Weyl's, 1952, p. 132, understanding that "objectivity means invariance with respect to the group of automorphisms"). We could then abstractly conceive of a state $\mathrm{w}$ of $W$ (presumably at time $t$ ) as the entirety of all corresponding 'partial' states of the universe at time $t$, be it quantum-physical states, states of the sun, or states of human beings. However, HSP seem to have a different conception of 'objective world' in mind. This follows, in particular, from the notions $X=W$, or $X \subset W$, by which they define certain 'realist strategies,' which would not make sense on the above interpretation (because each $x \in X$ would in these cases be identical to a specific world state $w$, which by definition comprises aspects far outside potential human experience). The notions $X=W$, or $X \subset W$ are supposed to capture the idea that all or some perceptions are "true of the world.". However, 'truth' can usually only be assigned to items such as propositions, not to elements of the 'objective world' themselves. Strangely enough, the cases $X=W$, or $X \subset W$ would imply that some or all elements of $W$, i.e., 'world states' themselves, are 'true', which could suggest that HSP take the 'objective world' to consists of entities, such as propositions, to which truth values can be assigned. Indeed, some locutions in the paper (and also standard Bayesian formulations) suggest that $W$ and $X$ are interpreted as consisting of expressions that can be regarded as descriptions of states of the 'objective world', and states of 'experiences,' respectively. But if $W$ is regarded as a "fundamentally unknown world," the nature of such descriptions would remain obscure.

Furthermore, from the given definitions of $W$ and $X$, I find it incomprehensible how a relation between the two can be of the type 'correspondence,' 'being caused by,' or 'representing.' This applies, again, in particular for the cases $X=W$, or $X \subset W$. Locutions such as "correspondence between our representations and the objective world" or a "state $w \in W$ causes at most one perceptual experience $x \in X$ ", or "perceptions represent true structures in the objective world" are, without additional explications, unwarranted by and unmotivated within the formal framework outlined.

So, whichever way one looks at it, the conceptualizations of $W$ and $X$, as given by HSP, and the alleged relations between $X$ and $W$ do not, in my view, yield a coherent framework. Unless we can precisely determine the respective kinds of entities of which the sets $W$ and $X$ consist, a mathematical idealization of $W$ and $X$ as a measurable spaces hovers, as it were, in the air. Thus, the validity of HSP's conclusions hinges in particular on the question whether there is a coherent and precise notion of 'objective world' appropriate for their purposes, on the basis of which their formal analyses can warrant the conclusion HSP want to draw. It also has to be made clear why and in what sense the set $W$, which in some cases is identified, entirely or partly, with the set of 'perceptions', carries the attribute 'objective.'

I will now look at the premises of HSP from a more general point of view. In our ordinary discourse we usually have, of course, no problem in understanding, in some communicative context, what might be meant by the expression 'objective world.' We usually take the 'objective world' to be the entirety of things that actually exist and for which we have no reasons to assume that they are merely a product of our mental activities; our 'objective world' is hence populated by various kinds of everyday manifest objects, such as fleas, stars, clouds, rivers, or people. Our commonsense conception denies however this status to objects that are postulated by explanatory theories of physics, such as bosons, fermions, or quarks. In physics, in contrast, we could regard, in metatheoretical discourse, the 'objective world' as consisting of all those entities that are postulated by our currently best explanatory accounts of physics (which would, of course, make membership time-dependent).

In philosophy, on the other hand, we can find any number of metaphysical conceptions of what 'objective reality' is presumed to be. The same observation holds for the philosophy of physics. To mention just a few examples: Poincaré (1907, p. 14) held that "what we call objective reality is, in the last analysis, [...] the harmony expressed by mathematical laws. It is this harmony then which is the sole objective reality, the only truth we can attain." Russell, in his later work, argued that events, not particles, must be the 'stuff'of the ultimate material of the objective world (and that 'substance' is merely a convenient way of collecting events into bundles). The astrophysicist A.S. Eddington, in his The Nature of the Physical World, held that "the stuff of the world is mind-stuff" (and hasted to add "that by 'mind' I do not exactly mean mind and by 'stuff' I do not at all mean stuff').

Helmholtz (1883, p. 656) took the view that we know nothing about the 'real world' ("über das eigentlich Reelle") underlying our perceptions. All the same, "when different perceptions offer themselves to us, we are justified in inferring that the underlying real conditions are different." Among the points of view developed in the philosophy of science, the arguably most defensible forms of a scientific realism were instigated by ideas of Poincaré and Russell, and led to varieties of what is called 'structural realism.' Corresponding positions basically claim that all we can know about the 'objective world' is the structure of the relations between things and 
not the things themselves, or, in more radical variants, that there are no 'things' and that structure is all there is. While structural realism appears to be particularly fruitful when dealing with certain meta-theoretical problems of the natural sciences (e.g., Maxwell, 1970; Worrall, 1989), attempts at a coherent formulation are still encumbered with severe problems (e.g., Ainsworth, 2009). Nevertheless, it might at first sight be tempting to regard structural realism as an attractive option in perceptual psychology, because it would allow us to cling to our built-in realist convictions, while such a framework at the same time would avoid problems that are all too obvious in attempts to relate our 'perceptions' to the 'objective world.' Such a view would, however, misconstrue what is at stake in perception theory. For reasons indicated above, issues of structural realism have no bearing on perception theory, which deals with the identification of the internal principles of a specific biological system.

The theoretical clarifications achieved during the last centuries have clearly revealed that the notion of 'objective world' is of no relevance for the explanatory purposes of perception theory, and, in fact, borders on the inapprehensible, once we go beyond the context of ordinary discourse. This also applies to another fundamental notion of HSP's approach, namely 'truth,' whose meaning HSP likewise take to be selfevident. However, 'truth' is a notion that either belongs to ordinary discourse, with multifarious meanings that can vary with intentions and concerns, or belongs to metaphysical and philosophical discourse, where its meaning is notoriously elusive. As Horwich (1992, p. 510) noted: "The ancient idea that truth is some sort of 'correspondence with reality' has still never been articulated satisfactorily: the nature of the alleged 'correspondence' and the alleged 'reality' remain objectionable obscure." Fortunately, these difficulties are of no relevance in the context of perception theory, where we aim at a theoretical understanding of the internal principles of a biological system. For similar reasons, metaphysical notions such as 'truth' are of no explanatory avail when we attempt to understand the biological evolution, or the evolutionary dynamics of alleged 'evolutionary strategies' of the perceptual system.

Another crucial notion in HSP's analyses is that of natural selection, which they apparently and without further discussion regard as an almost exclusive factor regulating evolutionary change. In fact, however, the actual role of natural selection in the evolution of complex biological systems is far from obvious (cf., for example, Lewontin, 1972; Lynch, 2007). Evolutionary biology has, in more recent years, accumulated pervasive evidence that suggests that the vast majority of evolutionary change has rather little to do with natural selection. Accordingly, Lynch (2007, p. 368) contends that the "blind worship of natural selection is not evolutionary biology. It is arguably not even science. Natural selection is just one of several evolutionary mechanisms [...]." In the specific case of the perceptual system, it seems to be a reasonable conjecture that its core structural and computational properties will most likely be essentially co-determined and shaped, within the apparently rather broad latitude of design options that are left open by global adaptational restrictions, by powerful internal constraints, such as physical, architectural, or computational constraints, that arise during the evolutionary development of a system of this complexity (see Mausfeld, 2011, for some corresponding speculations). A proper account of the actual achievements of the perceptual system should make clear that these achievements go far beyond those that can be accounted for by effects of fitness and adaption. But whatever explanatory role might be assigned to natural selection in evolutionary biology, I know of no arguments from evolutionary biology that support HSP's claim that "the distinction between fitness and truth is central to evolutionary theory." Rather, 'truth' is a notion that, for principled reasons, cannot be expected to play a role in explanatory frameworks of evolutionary biology.

The value of simulation studies and formal analyses in the natural sciences crucially depends on the degree to which the underlying premises are empirically warranted, and on whether the idealizations employed are sufficiently constrained by the empirical domains under scrutiny. Watamull and Hauser (2014) remind us that "dynamic computational simulations generate insights insofar as the simulated system is rigorously defined in an abstract mathematical theory." This also holds for enquiries into the nature of the perceptual system. If the idealizations employed are not motivated and guided by a sufficiently rich body of substantial constraints derived from empirical research, the conclusions obtained from such studies cannot be expected to teach us anything about the actual internal principles on which the achievements of the perceptual system are based, or about the actual principles through which these principles have emerged evolutionarily from simpler sensory systems. Rather, they would carry the risk of distracting us from the serious and deep theoretical questions with which we have to grapple in perception theory.

Needless to say, in certain contexts of ordinary discourse, the general question that seems to motivate HSP's endeavour, namely whether perception mirrors the 'true structure of the objective world,' can be a meaningful and sensible one. Such a question, however, will hardly survive the transition into a natural science context. It rather seems that no question remains that can be posed in a coherent and intelligible way. Hence, the appropriate response to such a question is not to evaluate specific proposals but rather to dispel the delusion that an intelligible question has been raised.

Just like other domains of the natural sciences, perception theory has to disentangle itself from metaphysical issues, such as those pertaining to 'truth' or 'correspondence to the objective world,' and, more generally, has to divest its theoretical 
notions of the distorting residues of commonsense intuitions. Nevertheless, sometimes aspects of our ordinary intuitions can, as the history of science shows, partly be captured by much narrower questions that are amenable to scientific enquiry. This also holds for questions about how specific achievements of the perceptual system are related to regularities of its environment, or to its Uexküllean 'Umwelt,' and how relevant regularities can be appropriately described in physical or mathematical terms. In any case, such questions pertain to a different level of analysis than the one of perception theory proper, which aims at a theoretical understanding of the internal principles of a specific biological system. In biology, it is regarded as a matter of course that relevant phenomena can and have to be investigated on separate levels of analysis, such as function, physical basis, and evolutionary development. When we go beyond perception theory proper, we may ask all sorts of questions concerning different levels of analysis, pertaining to, for example, how the perceptual system is embedded into the internal functional architecture of the mind/ brain; how its achievements are tied to specific aspects of its environment; or what constitutes the physical basis of its computational apparatus (e.g., questions concerning the role of proteins as computational devices). Whatever aspect we are interested in, there is no reason to expect that notions such as 'truth' or 'correspondence to the objective world' play an explanatory role in a scientific context.

\section{References}

Ainsworth, P. M. (2009). Newman's objection. British Journal for the Philosophy of Science, 60, 135-171.

Brett, G. S. (1921). A history of psychology (Vol. 3). London: Allen \& Unwin.

Chomsky, N. (2000). New horizons in the study of language and mind. Cambridge: Cambridge University Press.

Helmholtz, H. V. (1883). Wissenschaftliche Abhandlungen, Bd. 2. Leipzig: Barth.

Hoffman, D. D., Singh, M., Prakash, C. (2015). The interface theory of perception. Psychonomic Bulletin \& Review, in press.

Horwich, P. (1992). Theories of truth. In J. Dany \& E. Sosa (Eds.), A companion to epistemology (pp. 509-515). Oxford: Blackwell.
King, P. (1994). Scholasticism and the philosophy of mind: The failure of Aristotelian psychology. In T. Horowitz \& A. Janis (Eds.), Scientific failure (pp. 109-138). Lanham: Rowman and Littlefield.

King, P. (2008). The inner cathedral: Mental architecture in high scholasticism. Vivarium, 46, 253-274.

Lewontin, R. (1972). Testing the theory of natural selection. Nature, 236, $181-182$.

Lynch, M. (2007). The origins of genome architecture. Sunderland: Sinauer.

Mausfeld, R. (2002). The physicalistic trap in perception theory. In D. Heyer \& R. Mausfeld (Eds.), Perception and the physical world (pp. 75-112). Chichester: Wiley.

Mausfeld, R. (2010a). The perception of material qualities and the internal semantics of the perceptual system. In L. Albertazzi, G. van Tonder, \& D. Vishwanath (Eds.), Perception beyond inference. The information content of visual processes (pp. 159-200). Cambridge: MIT Press.

Mausfeld, R. (2010b). Colour within an internalist framework: The role of 'colour' in the structure of the perceptual system. In J. Cohen \& M. Matthen (Eds.), Color ontology and color science (pp. 123-148). Cambridge: MIT Press.

Mausfeld, R. (2011). Intrinsic multiperspectivity. Conceptual forms and the functional architecture of the perceptual system. In W. Welsch, W. J. Singer, \& A. Wunder (Eds.), Interdiciplinary anthropology: Continuing evolution of man (pp. 19-54). Berlin: Springer.

Mausfeld, R. (2012). On some unwarranted tacit assumptions in cognitive neuroscience. Frontiers in Cognition, 3(67), 1-13. doi:10.3389/ fpsyg.2012.00067

Mausfeld, R. (2013). The attribute of realness and the internal organization of perceptual reality. In L. Albertazzi (Ed.), Handbook of experimental phenomenology. Visual perception of shape, space and appearance (pp. 91-118). Chichester: Wiley.

Maxwell, G. (1970). Theories, perception, and structural realism. In R. G. Colodny (Ed.), The nature and function of scientific theories (pp. 3 34). Pittsburgh: University of Pittsburgh Press.

Meier-Oeser, S. (1997). Die Spur des Zeichens. Das Zeichen und seine Funktion in der Philosophie des Mittelalters und der frühen Neuzeit. Berlin: De Gruyter.

Poincaré, H. (1907). The value of science. New York: The Science Press.

Tachau, K. (1988). Vision and certitude in the age of Ockham: Optics, epistemology and the foundations of semantics, 1250-1345. Leiden: Brill.

Watumull, J., \& Hauser, M. D. (2014). Conceptual and empirical problems with game theoretic approaches to language evolution. Frontiers in Psychology, 5, 226. doi:10.3389/fpsyg.2014.00226

Weyl, H. (1952). Symmetry. Princeton: Princeton University Press.

Worrall, J. (1989). Structural realism: The best of both worlds? Dialectica, 43, 99-124.

Yolton, J. W. (1984). Perceptual acquaintance from Descartes to Reid. Minneapolis: University of Minnesota Press.

Yolton, J. W. (1996). Perception and reality. A history from Descartes to Kant. Ithaka: Cornell University Press. 